\title{
Pre-clinical murine models: syngeneic models for immuno-oncology
}

\begin{abstract}
Experimental animal models are crucial in the study of the pathological development of cancer and evaluation of the efficacy of novel therapeutic or preventive agents. To date, several newer targeted therapeutics has been unsuccessful. Novel therapeutics that has often appeared to perform well in preclinical models has failed in the clinic. Many factors contribute to these failures, but the one most often attributed is the short-comings of the preclinical models. Several animal models are available including a wide variety of genetic mouse models, cell line xenografts, patient derived xenografts, and syngeneic xenografts. These models have generated an enormous amount of information useful for the understanding of cancer. The complexities and strategies of these models is beyond the scope of this mini-review, the aim here is to briefly discuss the strengths and weaknesses of some of the popular models. Given the progress in the rapidly developing field of immuno-oncology, particular emphasis will be placed on the potential for syngeneic murine models as a means of testing novel immune-modulatory compounds. New and improved preclinical mouse models, combined with technological advances to study such models, will undoubtedly render the success of future human clinical trials for cancer patients.
\end{abstract}

Volume 2 Issue 4 - 2015

Joseph F Murphy

Charles River Laboratories, USA

Correspondence: Joseph F Murphy, Cancer Therapeutics and Immunology, Southern Research Institute, 10 Bursley Place, White Plains, New York 10605, USA, Tel 205-500-8990, Email joseph.murphy@crl.com

Received: August 21, 20I5 | Published: September 28, 2015
Abbreviations: SCID, severe combined immunodeficiency; PDX, patient-derived xenografts; NK, natural killer; CTLA-4, T-Lymphocyte-associated antigen-4; PD-1, programmed death; PDL1, programmed death ligand; Treg, T-regulatory (Treg); MDSC, myeloid-derived suppressor cells

\section{Introduction}

Preclinical murine models are essential in the drug discovery and development process for new cancer drugs, small molecules and biologics. In spite of this, they are imperfect copies of human cancers given their genetic and epigenetic heterogeneity and the multiplicity of dysregulated survival and growth-regulatory pathways that characterize the disease. ${ }^{1}$ This mini-review discusses some of these preclinical animal models for cancer with respect to their advantages, limitations, physiological and pathological relevance, and how they can best be utilized to evaluate the efficacy of novel therapeutic agents emerging from drug discovery programs.

\section{Preclinical murine models}

Preclinical murine models of cancer, including xenografts, genetically engineered, and syngeneic mice, have been developed as a means of studying the development and progression of the disease. Such models help to increase our understanding of the etiology and dissemination of cancer to overcome barriers to early detection and resistance to standard chemotherapy. ${ }^{2,3}$ Some of these methods have been used for decades and are well-established models; others are more recent and continue to develop. Animal models have been instrumental in elucidating key biochemical and physiologic processes of cancer onset and propagation in a living organism. Most importantly, they have served as a surrogate for patients in the evaluation of novel diagnostic and therapeutic anticancer drugs. ${ }^{4}$ Improvements have led advances in the field, such as live animal imaging techniques, allow for effective monitoring of the microenvironment and therapeutic efficacy. ${ }^{2}$

\section{Xenograft models}

One of the most widely used models is the human tumor xenograft.
Here, human tumor cells are transplanted, either under the skin or into the organ type in which the tumor originated, into immunocompromised mice that do not reject human cells. For example, the xenograft will be readily accepted by athymic nude mice, severely compromised immuno-deficient (SCID) mice, or other immuno-compromised mice. ${ }^{5}$ The development of human tumor xenograft models was a big step in moving toward more clinically relevant tumor models. ${ }^{6,7}$ The advantages of using human tumor xenografts are:

- Malignant cells are human.

- Reproducibility of many of the models.

- Long history and a strong baseline of drug response data.

- Readily available hosts.

- Statistically valid numbers of mice can be used in studies.

- Availability of a wide variety of tumor lines.

Some disadvantages of using these models:

- Syngeneic models are less costly to run.

- Stromal component of the tumors is rodent.

- Most of the tumor lines were developed using early technology.

- Tumors are regularly grown in a non-natural site (subcutaneous).

However, the major disadvantage is the lack of immune response inherent in these models. Nude mice are athymic and, therefore, have a limited $\mathrm{T}$ cell response, and severe combined immunodeficiency (SCID) strains lack both $\mathrm{T}$ and $\mathrm{B}$ cell responses. ${ }^{2}$ Because tumors can promote anti-tumor responses, such as tumor infiltrating lymphocytes, macrophages and myeloid-derived immuno-suppressor cells (MDSCs), these models may not accurately represent disease progression and therapeutic response observed in otherwise immunecompetent individuals. ${ }^{8,9}$ As a result of these inherent weaknesses, alternative models have been utilized, emphasizing a need for improvements to current models. ${ }^{2}$ 


\section{PDX models}

Another option in addition to traditional cell line-derived xenograft models involves the direct transfer of tumor fragments from individual patients. Tumor graft models (otherwise referred to as patient-derived xenografts, PDX) are based on the transfer of primary tumors directly from the patient into an immuno-deficient mouse. ${ }^{3}$ Because PDX mice are derived from human tumors, they offer a tool for developing anticancer therapies and personalized medicine and can also be used to study metastasis and tumor genetic evolution. In spite of these advantages, several factors have contributed to hinder the use of PDX mice. The first is cost, tumor grafts can only be maintained in mice, and their passage requires a more specialized skill set than does the simple maintenance of cultured cell lines. Moreover, PDX models can suffer from long latency periods after engraftment and variable engraftment rates. Tumor graft latency, measured as the time between implantation and the development of a progressively growing xenograft tumor, can range from 2 to 12 months. ${ }^{10,11}$ Engraftment rates typically vary depending on the tumor type. Higher engraftment rates are associated with more clinically aggressive tumors. ${ }^{12}$ Correlations between poor prognosis and engraftment rate were so marked that it has been suggested to be predictive of the disease course. ${ }^{13}$ Finally, there is the problem of broad availability and the number of PDX models that have been reported. ${ }^{14}$ Newer strategies to build orthotopic models include implanting human bone marrow-derived mesenchymal stem cells resulting in greater vascularity and maintenance of hormonal status. ${ }^{15}$

\section{Syngeneic tumor models}

Allograft mouse tumor systems, otherwise known as syngeneic or syngeneic models, are tumor models whose genetic background is similar, if not identical, to the host animal. ${ }^{16}$ Because they retain intact immune systems, these models can be particularly pertinent for studies of immunologically-based targeted therapies, either used alone or in combination. Lack of rejection of the transplant by the host's immune system allows researchers to monitor the tissues for changes, such as growth or shrinkage, metastasis, and survival rate. Therapeutic interventions can be performed and the results are assessed to understand the treatment potentials. A mouse tumor growing in mice of the strain in which the tumor originated, offer several advantages. ${ }^{17}$ These advantages include:

i. Relatively low cost and high reproducibility.

ii. Grow in immunocompetent hosts.

iii. Wide variety of tumor types.

iv. Generally non-immunogenic.

v. Long history of use and strong baseline of drug response data.

vi. Hosts are readily available.

vii. Studies are easily conducted with statistically meaningful numbers of mice per group.

The main disadvantages of syngeneic tumor models are that the tumor cells are rodent, and therefore express the mouse/rat homologues of the desired targets, and the tumors tend to grow fast. In spite of this, syngeneic models are proving to be an excellent model to test compound focusing on immuno-oncology targets for the treatment of cancer.

\section{Syngeneic models and immuno-oncology}

The immune system has the greatest potential for the specific destruction of tumors with no toxicity to normal tissue and for longterm memory that can prevent cancer recurrence. The last 30 years of immuno-oncology research have provided solid evidence that tumors are recognized by the immune system and their development can be stopped or controlled long term through a process known as immuno-surveillance. ${ }^{18}$ In many cancers, malignant progression is accompanied by profound immune suppression that interferes with an effective anti-tumor response and tumor elimination. Initially, most of the escape from immuno-surveillance was ascribed to changes in the tumor cells themselves (loss of tumor antigens, loss of human leukocyte antigen molecules, loss of sensitivity to complement, or T-cell or natural killer (NK) cell lysis), making them a poor target of an immune attack. However, it has become clear that the suppression comes from the ability of tumors to subvert normal immune regulation to their advantage. The tumor microenvironment can prevent the expansion of tumor antigen-specific helper and cytotoxic T-cells and instead promote the production of pro-inflammatory cytokines and other factors, leading to the accumulation of suppressive cell populations that inhibit instead of promote immunity. ${ }^{18}$

\section{Checkpoint inhibitors}

Checkpoint inhibitors represent the most heralded drugs in immuno-oncology oncology. They work by removing the brake on the immune system to promote its activation. In other words they block the molecules that switch off immune cells. The best described are regulatory T-cells and myeloid-derived suppressor cells. Great conceptual and technical advances in the field of immuno-oncology over the past 30 years have provided us with the knowledge and techniques to develop novel immunotherapeutic approaches for the treatment of cancer. These include methods that enhance tumor immunity by blocking inhibitory pathways and inhibitory cells in the tumor microenvironment, for example, antibodies against cytotoxic T-lymphocyte-associated antigen-4 (CTLA-4), programmed death 1 (PD-1) or its ligand programmed death ligand 1 (PDL-1). Similarly, they include methods that can enhance the specificity of anti-tumor immunity by inducing the expansion of $\mathrm{T}$ cells and antibodies directed to well-defined tumor antigens, examples include cancer vaccines, potent adjuvants, immuno-stimulatory cytokines. Even as monotherapies, these approaches have a substantial impact on the treatment of some patients with advanced, previously untreatable, malignancies. These successes provide a rationale to expect that used in various combinations or earlier in the disease, current and future immunotherapy can transform cancer treatment, improving a prognosis for many patients.

One direction is to continue using the old classes of immunotherapy that target the cancer directly, but to use them in combination with therapies that target the immune system in the tumor microenvironment, such as cytokines, suppressors of T-regulatory (Treg) or myeloid-derived suppressor cells (MDSC) activity, or antibodies that modulate T-cell activity. The recently approved antibody, ipilimumab, which acts to sustain cytotoxic T-cell activity by augmenting T-cell activation and proliferation, is one example of such an immuno-modulatory agent. ${ }^{19}$

Another direction is to use immunotherapy, both old and new, for preventing cancer in individuals at high risk. ${ }^{20}$ Studies of the tumor microenvironment are providing information about immunosurveillance of tumors from early premalignant lesions to more advanced dysplastic lesions to cancer. At each step, tumor-derived and immune system-derived components have a unique composition that will have distinct effects on immunotherapy. Because 
these premalignant microenvironments are less developed and immunosuppressant is less entrenched, it should be easier to modulate towards the elimination of abnormal cells. For a comprehensive review of existing and future directions in cancer immunotherapy, see review. ${ }^{21}$

\section{Conclusion}

Enormous strides have been made cancer therapy over the last few years. Currently, the field of immuno-oncology has emerged as one of the hottest areas in drug discovery, development, and clinical translation. Some of the newest approaches with checkpoint inhibitors, cancer vaccines, $T$ cell therapies, among others, have provided clinical efficacy in many patients. With a conservative prediction of 500 new clinical trials to begin in 2016, there is still more to do to bridge the gap between patients that respond and do not respond to treatment. Central to this effort will be the use of appropriate preclinical models. In addition to the afore-mentioned models, humanized mice, produced through inoculating human hematopoietic cells into immunocompromised mice or creating transient human immunity by mixing human peripheral blood mono-nucleated cells with xenograft models, will likely play a prominent role. For now though, the spotlight is on syngeneic models, and these will likely form the backbone of many preclinical immuno-oncology programs, particularly with the increasing focus on combination therapy. Syngeneic models will remain a valuable source of in vivo information and will play a prominent role in elucidating potential compounds, either alone or in combination, that demonstrate efficacy as immunotherapeutic anticancer agents.

\section{Acknowledgements}

None.

\section{Conflicts on interest}

The author declares that there is no conflict of interest.

\section{Funding}

None.

\section{References}

1. Ruggeri BA, Camp F, Miknyoczki S. Animal models of disease: preclinical animal models of cancer and their applications and utility in drug discovery. Biochem Pharmacol. 2014;87(1):150-161.

2. House CD, Hernandez L, Annunziata CM. Recent technological advances in using mouse models to study ovarian cancer. Front Oncol. 2014;4:26.

3. Siolas D, Hannon GJ. Patient-derived tumor xenografts: transforming clinical samples into mouse models. Cancer Res. 2013;73(17):53155319 .
4. DeJong M, Maina T. Of mice and humans: are they the same? Implications in cancer translational research. J Nucl Med. 2010;51(4):501-504.

5. Richmond A, Su Y. Mouse xenograft models vs GEM models for human cancer therapeutics. Dis Model Mech. 2008;1(2-3):78-82.

6. Kelland LR. "Of mice and men": values and liabilities of the athymic nude mouse model in anticancer drug development. Eur $J$ Cancer. 2004;40(6):827-836.

7. Sausville EA, Newell DR. Preclinical models in cancer drug discovery and development. Eur J Cancer. 2004;40:783-784.

8. Anderson MJ, Shafer-Weaver K, Greenberg NM, et al. Tolerization of tumor-specific $\mathrm{T}$ cells despite efficient initial priming in a primary murine model of prostate cancer. J Immunol. 2007;178(3):1268-1276.

9. Gavalas NG, Karadimou A, Dimopoulos MA, et al. Immune response in ovarian cancer: how is the immune system involved in prognosis and therapy: potential for treatment utilization. Clin Dev Immunol. 2010.

10. Dangles-Marie V, Pocard M, Richon S, et al. Establishment of human colon cancer cell lines from fresh tumors versus xenografts: comparison of success rate and cell line features. Cancer Res. 2007;67(1):398-407.

11. Bergamaschi A, Hjortland GO, Triulzi T, et al. Molecular profiling and characterization of luminal-like and basal-like in vivo breast cancer xenograft models. Mol Oncol. 2009;3(5-6):469-482.

12. Zhao X, Liu Z, Yu L, et al. Global gene expression profiling confirms the molecular fidelity of primary tumor based orthotopic xenograft mouse models of medulloblastoma. Neurooncology. 2012;14:574-583.

13. DeRose YS, Wang G, Lin YC, et al. Tumor grafts derived from women with breast cancer authentically reflect tumor pathology, growth, metastasis and disease outcomes. Nat Med. 2011;17(11):1514-1520.

14. Marangoni E, Vincent-Salomon A, Auger N, et al. A new model of patient tumor-derived breast cancer xenografts for preclinical assays. Clin Cancer Res. 2007;13(13):3989-3998.

15. DeRose YS, Gligorich KM, Wang G, et al. Patient-derived models of human breast cancer: protocols for in vitro and in vivo applications in tumor biology and translational medicine. Curr Protoc Pharmacol. 2013.

16. Barnes S. The rise of syngeneic models in cancer immunotherapy. Drug Discovery \& Development. 2015.

17. Sporn MB, Liby KT. Cancer chemoprevention: scientific promise, clinical uncertainty. Nat Clin Pract Oncol. 2005;2(10):518-525.

18. Finn OJ. Immuno-oncology: understanding the function and dysfunction of the immune system in cancer. Ann Oncol. 2012;23(suppl 8).

19. Callahan MK, Wolchok JD, Allison JP. Anti-CTLA-4 antibody therapy: immune monitoring during clinical development of a novel immunotherapy. Semin Oncol. 2010;37(5):473-484.

20. Lollini PL, Cavallo F, Nanni P, et al. Vaccines for tumour prevention. Nat Rev Cancer. 2006;6:204-216.

21. Murphy JF. Oncology discovery center of excellence strengthened by addition of immunotherapy researchers. Springer. 2015. 